Article

\title{
One-Step Preparation of Phenyl Boron-Modified Magnetic Mesoporous Silica for Selective Enrichment of cis-Diol-Containing Substances
}

\author{
Hua Fu ${ }^{1,2}$, Jing $\mathrm{Hu}^{1}$, Min Zhang ${ }^{3, *}$, Yuerong Wang ${ }^{1}$, Hongyang Zhang ${ }^{1}$ and Ping $\mathrm{Hu}{ }^{1, *}$ (D) \\ 1 Shanghai Key Laboratory of Functional Materials Chemistry, School of Chemistry \& Molecular Engineering, \\ East China University of Science and Technology, Shanghai 200237, China; fuhua8711@126.com (H.F.); \\ hjhuali2012@163.com (J.H.); wangyuerong@ecust.edu.cn (Y.W.); hongyang_zhang@ecust.edu.cn (H.Z.) \\ 2 School of Chemical Engineering, Qinghai University, Xining 810016, China \\ 3 Shanghai Key Laboratory of New Drug Design, School of Pharmacy, East China University of Science \\ and Technology, Shanghai 200237, China \\ * $\quad$ Correspondence: zhangm@ecust.edu.cn (M.Z.); huping@ecust.edu.cn (P.H.); Tel.: +86-021-6425-2844 (M.Z. \& P.H.)
}

Received: 1 February 2018; Accepted: 2 March 2018; Published: 7 March 2018

\begin{abstract}
For enrichment and separation of cis-diol-containing compounds from biomatrix, a new type of magnetic nanoparticles named MS-48-PBSC, whichwas facilely prepared in a one-step heterogeneous reaction. The morphology results demonstrated that the MS-48-PBSC was a spherical nanomaterial containing a core of silica-coated magnetic particle with a diameter of about $200 \mathrm{~nm}$, and a cover layer of mesoporous silica with a thickness of approximate $50 \mathrm{~nm}$. The characterization results showed that MS-48-PBSC presented a pore size of $4.2 \mathrm{~nm}$, a surface area of $548 \mathrm{~m}^{2} \cdot \mathrm{g}^{-1}$, and a pore volume of $0.30 \mathrm{~cm}^{3} \cdot \mathrm{g}^{-1}$. The MS-48-PBSC also exhibited magnetism of $42 \mathrm{emu} \cdot \mathrm{g}^{-1}$ that contributed to the easy separation of magnetic nanomaterial within $30 \mathrm{~s}$ from the matrix with the aid of the external magnetic field. In addition, the MS-48-PBSC exhibited high adsorption capacity for adenosine, xanthosine, uridine, sialic acid, and teicoplanin with $0.60,0.51,0.42,0.75$, and $1.26 \mathrm{mg} / \mathrm{g}$, respectively, and showed a high selectivity for the cis-diol structure compounds, relative to interferences of bovine serum albumin, guanine, uric acid, and xanthine. The recoveries of adenosine, xanthosine, uridine, sialic acid, and teicoplanin were $71.8-114.1 \%$ with relative standard deviation (RSD) $\leq 8.6 \%$, and the enrichment factors of them were $8-11$. MS-48-PBSC exhibited quick separation capability from matrix, high adsorption capacity and size exclusion for bovine serum albumin, which could meet the requirements of separation and enrichment for substances with a cis-diol structure.
\end{abstract}

Keywords: magnetic solid-phase extraction; one-step preparation; phenylboronic acid; cis-diolcontaining substance; selective enrichment

\section{Introduction}

Cis-diol-containing biomolecules, such as sugars, nucleosides, and catecholamines, are closely related to physiological functions and diseases and participate in various biochemical reactions and life activities [1,2]. The identification of these molecules is of considerable significance. Molecules of medicine, including aminoglycoside antibiotics, flavonoid glycosides, and saponins from natural sources, also contain the cis-diol function group [3]. The biomolecules and drug metabolites are widely distributed in tissue, blood, and urine [4-6]. Some of these substances are present at trace levels in complex matrices and difficult to detect directly. Thus, pretreatment technologies for enriching target compounds with cis-diol structures and eliminating matrix interference should be developed. Boron-group-functionalized materials have become the main solid-phase extractants in the separation 
and enrichment of cis-diol compounds [7]. Given the boronic acid group is at equilibrium between the undissociated and dissociated forms by a changing $\mathrm{pH}$, the group can be used as selective adsorbent for cis-diol compounds. Under alkaline conditions, the boron atom is complexed with oxygen and changes from the original planar $s p^{2}$ hybrid to the tetrahedral $s p^{3}$ hybrid. The cis-boric acid ions simultaneously form a five- or six-membered cyclic ester with 1,2-cis-diols. When the condition becomes acidic, $s p^{3}$ hybridizes back to the $s p^{2}$ hybrid, and the cyclic ester dissociates [8-10]. Therefore, the separation and enrichment of cis-diol compounds from complex samples by using boron-affinity materials are highly selective and easy to operate.

To date, a variety of boron-affinity materials, such as boron-based polymer resins, molecularly imprinted materials, magnetic nanoparticles, silica particles, monolith columns, and graphenes have been developed. The materials have been widely applied in sample pretreatment, such as solid-phase extraction (SPE) and dispersive extraction-adsorption. Compared with other materials, the prominent feature of boron-based magnetic nanomaterials is that the solid-liquid separation can be simplified by applying a magnetic field. In addition, a higher extraction efficiency and faster desorption time can be achieved on nanoparticles compared with micron-sized particles due to the large surface area [11-13]. Xiong and coworkers [14] developed one type of boron-affinity magnetic nanoparticles. Ferroferric oxide magnetic nanoparticles, ethyl orthosilicate hydrolysates, and bifunctional-molecule-modified epoxy groups were used as core, cover layer, and surface functional groups, respectively. Amino phenylboronic acid was finally immobilized on the surface of the magnetic nanoparticles through a ring-opening reaction. This method utilized the easy modification of silica gel groups and thus became one of the most commonly used methods for the preparation of boron-affinity magnetic materials. The solvothermal method is another commonly used method for the preparation of magnetic nanoparticles by direct modification of specific active groups. Materials with specific active groups were subsequently directly grafted using boronic acid groups for the determination of biomolecules. Deng et al. [15] prepared amino-group-modified magnetic iron oxide nanoparticles by using the solvothermal method and then obtained the magnetic boron-affinity material by using a sequential two-step amide reaction with oxalyl chloride and amino phenylboronic acid. This magnetic boron-affinity material was suitable for glycoprotein separation. In recent years, in order to improve the extraction capacity of boron-based nanomaterials, researchers have increased the specific surface area of nanoparticles and the surface grafting rate of the functional group.

Magnetic mesoporous nanosilica (MMNS) materials contain a core of silica-coated magnetic practical and a cover layer of mesoporous nanosilica. As functional nanomaterials used for SPE, MMNS possesses an easily modifiable $\mathrm{Si}-\mathrm{OH}$ on the surface and uniform mesopore structure for carrying targets. Pore size of MMNS is also easily adjustable for various applications. We propose that modification of the mesoporous silica surface by using phenylboron to increase the loading capacity of boron-affinity materials. In addition, the exterior mesoporous silica shell can also keep the macromolecular interference (such as bovine serum albumin (BSA)) in the matrices out of the interior pore walls through the adjustable mesopore diameters. This method improves the selectivity of boron-affinity materials to the small-molecule cis-o-dihydroxy compounds.

The most common MMNS were prepared using MCM-41 and SBA-15 as their exterior shells. The former material contains an exterior mesoporous shell within a confined molecular pore size of 2.3-3.8 nm. Such small pore sizes were unfavorable for immobilizing phenylboronic acid groups onto the surface of the magnetic mesoporous layer. The SBA-15 contains a mesoporous pore size within $8-16 \mathrm{~nm}$. The pore size allowed phenylboronic acid groups to be immobilizated, but such large dimensions result in the absence of size exclusion capability for certain macromolecular compounds, such as chorionic gonadotropin $(36.7 \mathrm{kDa}, 7.5 \mathrm{~nm} \times 3.5 \mathrm{~nm} \times 3 \mathrm{~nm})$, luteinizing hormone (approximately $30 \mathrm{kDa}$ ), and a thyroid stimulating hormone (approximately $25-28 \mathrm{kDa}$ ). This drawback lead to inevitable interference on the selective adsorption for small-molecule cis-diol compounds.

For the modification of phenylboron functions on the silicon surface, the common method was to heterogeneously modify the phenylboron to the silicon material through multi-step heterogeneous 
reaction. However, the yield of phenylboron-based modification is low. Obviously, various factors including reaction kinetics would affect heterogeneous reactions. In order to meet the challenge, $\mathrm{Xu}$ et al. [8] repeated the grafting reaction twice to improve the yield of the boronic acid functionalized FDU-12 mesoporous material. $\mathrm{Xu}$ and coworkers [16] modified the polyethyleneimine on the silicon surface to increase the amount of modification of the boron group with a sequential four-step heterogeneous amidation reaction. However, the purification process of the product consumed a lot of solvent and time to clean and centrifuge the nanometer-sized product, which would easily lead to the loss of materials.

In our previous work, a facile method was developed to prepare magnetic mesoporous silica nanoparticles with an average pore size of $\sim 5.0 \mathrm{~nm}$ by using polyethylene glycol-polylactic acid copolymer as a template. Size exclusion for macromolecular compounds with a molecular weight of more than $20 \mathrm{kDa}$ can be performed on this nanomaterial [17]. If these magnetic mesoporous nanoparticles are used to immobilize a phenylboronic acid silane coupling (PBSC) agent onto the surface of a magnetic mesoporous layer, then the obtained new magnetic nanoparticles will have appropriate pore size for size exclusion, large surface area for loading capacity, and magnetic performance for simple process of separation of high selectivity and high capacity adsorption for small-molecule cis-diol compounds in a complex matrix.

To prepare boron-affinity materials, the phenylboron group is normally immobilized on the silicon material surface step-by-step by heterogeneous reaction. The low yield limited the application of this method. In this work, we aimed to develop a simple and convenient approach to preparation of boronic-modified MMNS named MS-48-PBSC based on a coupled reaction of a prepared PBSC agent with $\mathrm{Si}-\mathrm{OH}$ on the surface of MMSN. Then, we applied MS-48-PBSC to the adsorption and separation of small-molecule-containing cis-diol group.

\section{Results and Discussion}

\subsection{Synthesis of MS-48-PBSCP}

The preparation and application of MS-48-PBSC is illustrated in Scheme 1.

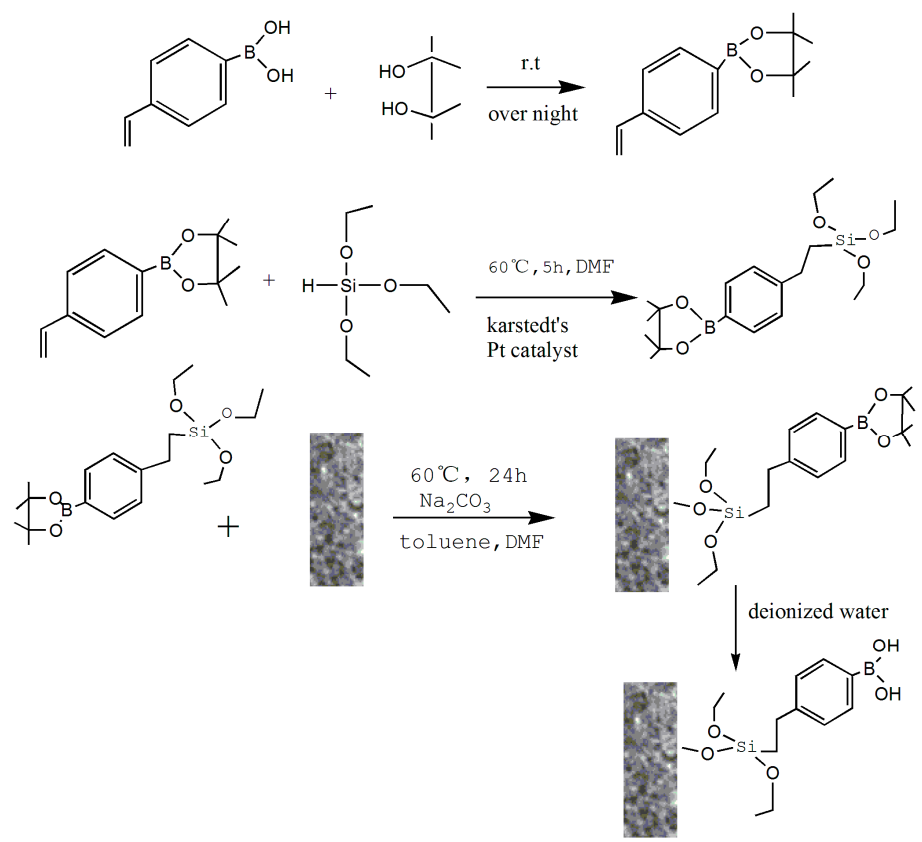

Scheme 1. Cont. 


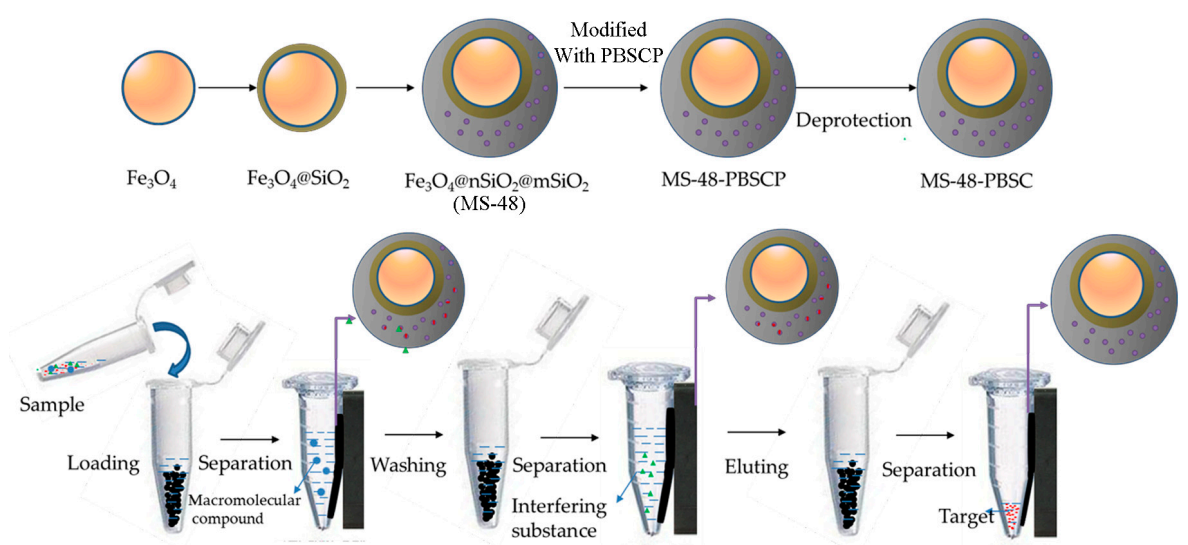

Scheme 1. Scheme of the preparation of mesoporous boronic acid-functionalized MS-48-PBSC and its application in magnetic solid-phase extraction. Target; macromolecular compound; $\Delta$ interfering substance; PBA, phenylboronic acid; MS-48-PBSCP, phenylboron modified magnetic mesoporous silica nanoparticles extractant.

Scheme 1 demonstrates the approach to preparation of MS-48-PBSC and its application process. First, we synthesized a silane-coupling agent PBSCP by hydrosilylation reaction with triethoxysilane and pinacol-protected 4-vinylbenzeneboronic acid as a starting reagent. The synthesis steps of PBSCP are shown at the top of the Scheme 1. Then, we applied the $\mathrm{Fe}_{3} \mathrm{O}_{4}$ particles as magnetic core and mesoporous material MS-48 as the outer cover, which has appropriate pore size with $4.7 \mathrm{~nm}$ to conjugate benzene boron silane coupling agent PBSCP. Finally, we used this new magnetic mesoporous material to extract and enrich several cis-diol-containing substances. The separation process is shown in the bottom of Scheme 1.

To prevent the introduced silane from affecting the stability of boron hydroxyl, we used a predesigned pinacol-protected 4-vinyl phenylboronic acid as an initial reactant to synthesize a benzene boron silane coupling agent with triethoxysilane through hydrosilylation reaction. Finally, MS-48-PBSCP was prepared using a coupled reaction that immobilized the PBSCP agent onto the surface of a magnetic mesoporous layer.

The product of PBSCP contained two substitution isomers, which can react with hydroxyl on MS-48. Hence, it is not necessary to separate them after the reaction. PBSCP was then grafted using a coupling reaction between triethoxysilane and $\mathrm{Si-OH}$ in one step. The first advantage of the coupling reaction is the extremely mild conditions and facile operation. The second advantage is that the one-step method significantly improved the synthetic efficiency for the heterogeneous reaction, In addition, the approach can reduce the posttreatment steps, which can not only make the product treatment simple but also reduce the usage of organic regents.

The infrared (IR) spectra (Figure 1a) of PBSCP and MS-48-PBSCP show bands centered at 2979, 2921 , and $1356 \mathrm{~cm}^{-1}$, which are ascribed to the vibrations of adsorbed $-\mathrm{CH}_{3}$ and $-\mathrm{CH}_{2}$. The vibration at $3429 \mathrm{~cm}^{-1}$ is ascribed to $-\mathrm{OH}$, and the vibration at $1611 \mathrm{~cm}^{-1}$ is ascribed to $\mathrm{C}=\mathrm{C}$ from the benzene rings. These results imply that the PBSCP-bonded molecules are successfully grafted onto the MS-48 materials [18].

The X-ray photoelectron spectroscopy (XPS) spectra (Figure 1b) of MS-48-PBSCP (top) and MS-48 (bottom) showed peaks centered at 714, 533, 285, 191, and $104 \mathrm{eV}$, which are the characteristic peaks of $\mathrm{Fe}, \mathrm{O}, \mathrm{C}, \mathrm{B}$, and $\mathrm{Si}$, respectively. The peak heights represent their relative content at the material surface. Evidently, MS-48-PBSCP displays a higher C peak than MS-48; the peak was derived from unhydrolyzed TEOS. The C and B content ratio in MS-48-PBSCP was in agreement with that of PBSCP, thus demonstrating the successful combination of PBSCP with the MS-48 surface. 

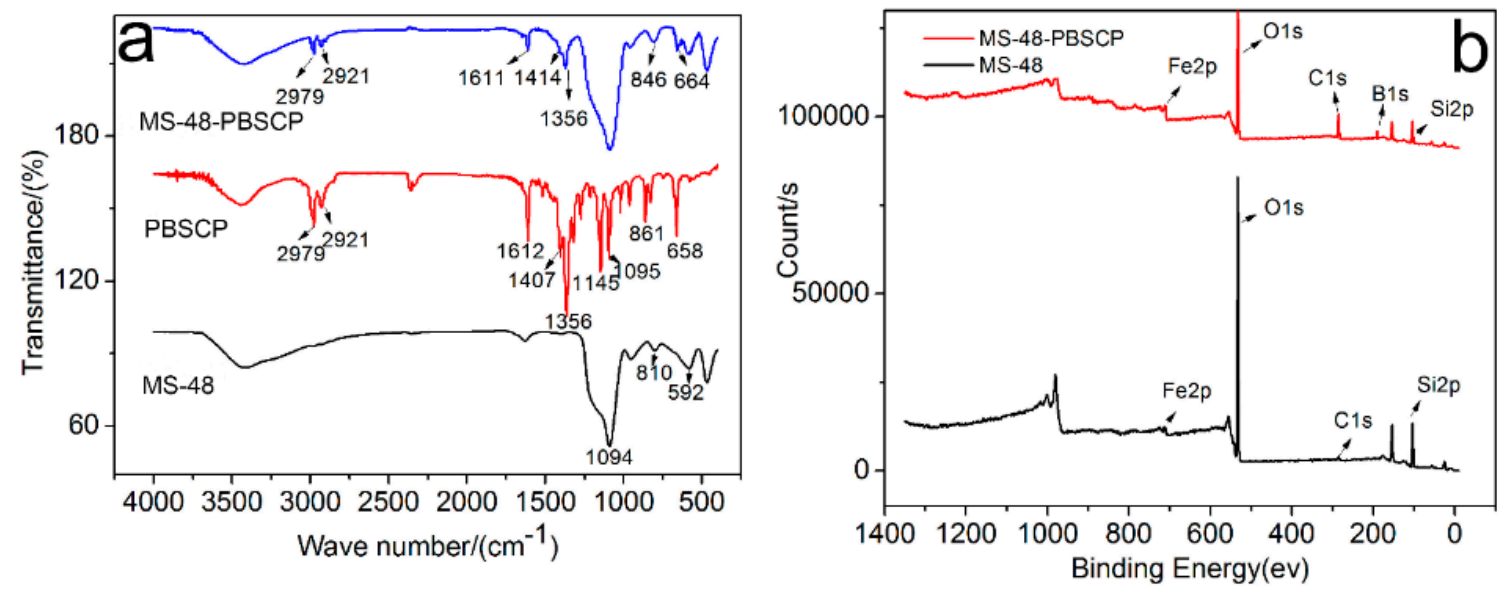

Figure 1. (a) Infrared spectroscopy of MS-48 (bottom), PBSCP (middle), and MS-48-PBSCP (top); (b) X-ray photoelectron spectroscopy curves of the mesoporous silica MS-48 and MS-48-PBSCP.

\subsection{Morphology of MS-48-PBSCP}

The surface profiles of MS-48 and MS-48-PBSCP are illustrated in Figure 2a,b. According to the scanning electron microscopy (SEM) images, the obtained microspheres exhibited a narrow size distribution with a mean diameter of $250 \mathrm{~nm}$, a spherical particle profile, uniform dispersion, and identical bubble size.

The transmission electron microscopy (TEM) images of MS-48 and MS-48-PBSCP (Figure 2a,b, respectively) show a magnetic core with a diameter of approximate $200 \mathrm{~nm}$ and porous silica shells with a thickness of approximate $50 \mathrm{~nm}$. The TEM images further show that the mesostructure is retained after functionalization. The pore sizes are approximate 4.7 and $4.2 \mathrm{~nm}$ for MS- 48 and MS-48-PBSCP, respectively, as derived from the adsorption branch by using the Barrett-Joyner-Halenda (BJH) method. MS-48-PBSCP exhibited a narrower pore size distribution than MS- 48 by approximate $0.5 \mathrm{~nm}$ (Figure 3). This result implied that the size exclusion was effective for molecules with a size of approximate $4 \mathrm{~nm}$, such as the hormone glycoproteins mentioned in the introduction. The Brunauer-Emmett-Teller (BET) surface area and total pore volume were $705 \mathrm{~cm}^{2} \cdot \mathrm{g}^{-1}$ and $0.38 \mathrm{~cm}^{3} \cdot \mathrm{g}^{-1}$ for MS- 48 and $548 \mathrm{~cm}^{2} \cdot \mathrm{g}^{-1}$ and $0.30 \mathrm{~cm}^{3} \cdot \mathrm{g}^{-1}$ for MS-48-PBSCP, respectively. This result implied that even after functionalization, the MS-48-PBSCP material retained its high porosity compared with MS-48. Moreover, the result suggested that the dense pore channels in the shell were exits to the microsphere surface, which improved the extraction capacity of the material.

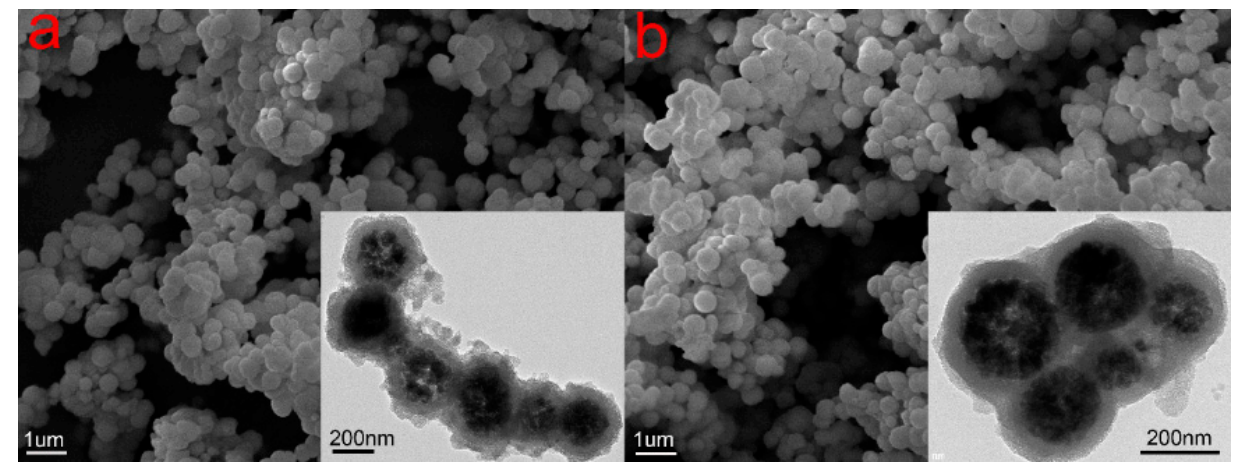

Figure 2. Scanning electron microscopy and Transmission electron microscopy (inset) images of (a) MS-48 and (b) MS-48-PBSCP. 


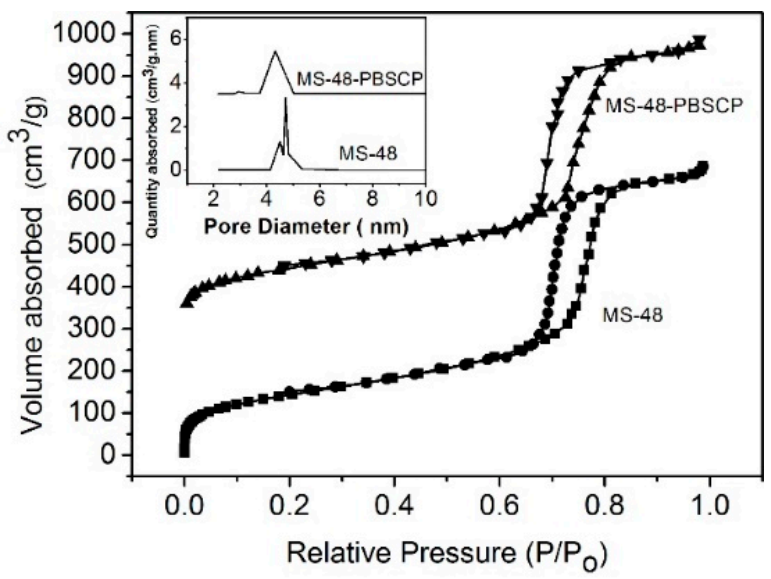

Figure 3. $\mathrm{N}_{2}$ sorption isotherm curves and pore size distribution plots for MS-48 and MS-48-PBSCP.

The saturation magnetization values of MS-48 and MS-48-PBSCP were approximate 44 and $42 \mathrm{emu}^{-1} \mathrm{~g}^{-1}$ (Figure 4). When suspended homogeneously in water, both MS-48 and MS-48-PBSCP exhibited fast sedimentation under the applied magnetic field in $30 \mathrm{~s}$. Hence, it was indicated that MS-48-PBSC had the fast separation performance.

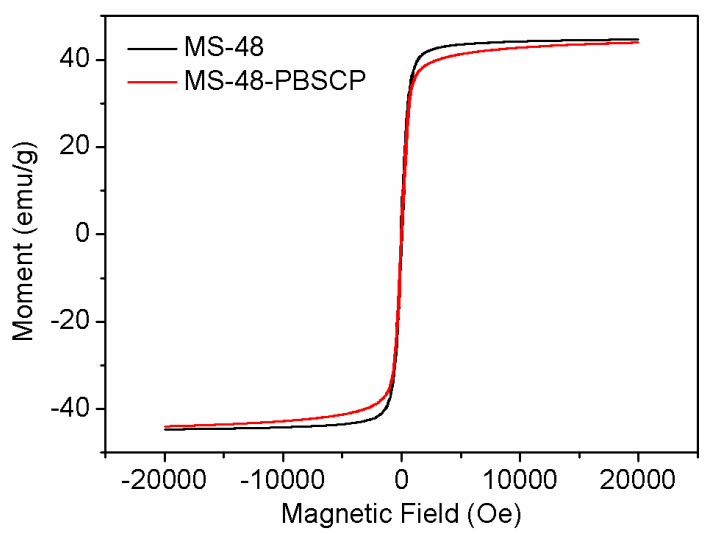

Figure 4. Room-temperature magnetic hysteresis loops of MS-48 and MS-48-PBSCP.

\section{3. $p H$ Investigation of Adsorption and Elution}

Binding $\mathrm{pH}$ is an essential factor for boronate to selectively adsorb or elute cis-diol structures. Boronate and cis-diol can complex to form a lactone structure under alkaline conditions and release cis-diols under acidic conditions. Notably, a low $\mathrm{pH}$ is a critical factor for the boronate affinity of cis-diol-containing biomolecules to ensure biological stability. The absorption capacities of MS-48-PBSC for adenosine at different $\mathrm{pH}$ values were therefore measured (Figure 5). Phosphate buffer saline (PBS) was used to adjust the $\mathrm{pH}$ in the 6.0-8.5 range, and hydrochloric acid-Tris buffer was used to adjust the $\mathrm{pH}$ in the 8.5 to 10.0 range. Upon the addition of adenosine (Figure 5a), the adsorption capacity during supernatant adsorption increased with increasing $\mathrm{pH}$. However, when the $\mathrm{pH}$ exceeded 7.8 , no evident change was observed. We therefore selected $\mathrm{pH}=7.8$ as the best binding $\mathrm{pH}$. The alkalescence adsorption condition indicated that MS-48-PBSC was fit for binding cis-diol-containing biomolecules, which usually exist in a neutral environment. During desorption (Figure 5b), we used formic acid from $\mathrm{pH} 1.0$ to 4.0 to elute the samples. Formic acid at $\mathrm{pH} 1.5$ produced optimum results. Therefore, we selected a formic acid solution with $\mathrm{pH}=1.5$ plus $40 \%$ methanol as the optimum elution conditions. 

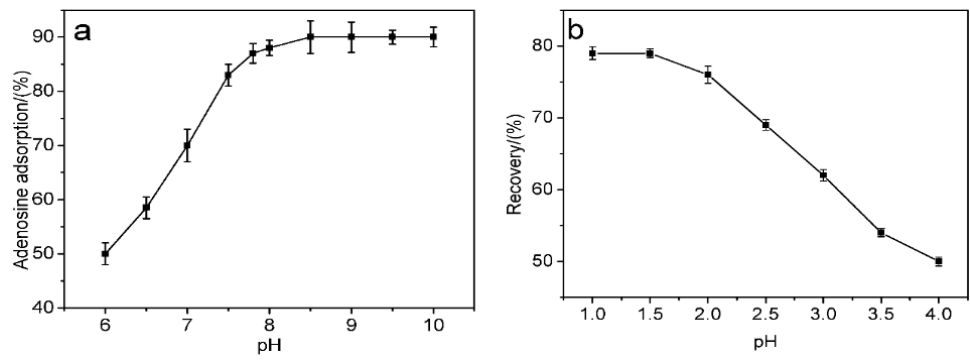

Figure 5. Effect of $\mathrm{pH}$ on MS-48-PBSC adsorption $(n=3)$. (a) Loading with adenosine in alkalinity buffer and (b) Eluting adenosine in acidic solution $(n=3)$.

\subsection{Adsorption Capacity Investigation}

We selected uridine, xanthosine, adenosine, sialic acid, teicoplanin as model compounds for nucleosides, monosaccharides and glycopeptide antibiotics in cis-diol-containing biomolecules to test the extraction performance of MS-48-PBSC. The adsorption capacity for targets was determined using high-performance liquid chromatograph (HPLC) and ultraviolet (UV)-visible (vis) spectrophotometry. All testing results are shown in Figure $6 \mathrm{a}-\mathrm{d}$. The loading time for the binding of uridine, xanthosine, adenosine, sialic acid, teicoplanin, and BSA to MS-48-PBSC was investigated. The results are shown in Figure $6 \mathrm{a}$. Figure $6 \mathrm{~b}$ shows the HPLC of elution of uridine, xanthosine, and adenosine with loading times of 2, 20, and $30 \mathrm{~min}$. Figure $6 \mathrm{c}$ shows the UV absorption spectrum of eluted sialic acid with loading times of 2, 20, and $30 \mathrm{~min}$, and Figure $6 \mathrm{~d}$ is the HPLC of eluted teicoplanin with loading times of 2, 20, and $30 \mathrm{~min}$. Following the addition of the tested samples to MS-48-PBSC, (Figure 6a-d), the absorption of all samples except for BSA rapidly increased and reached a maximum value within $30 \mathrm{~min}$. This result implied the rapid and stable bindings between cis-diols and MS-48-PBSC. Such characteristics are important features for high-throughput detection of cis-diol structures. The maximum adsorption amount of MS-48-PBSC reached 0.60, 0.54, 0.42, 0.75, and $1.26 \mathrm{mg} \cdot \mathrm{g}^{-1}$ for adenosine, xanthosine, uridine, sialic acid, and teicoplanin, respectively.
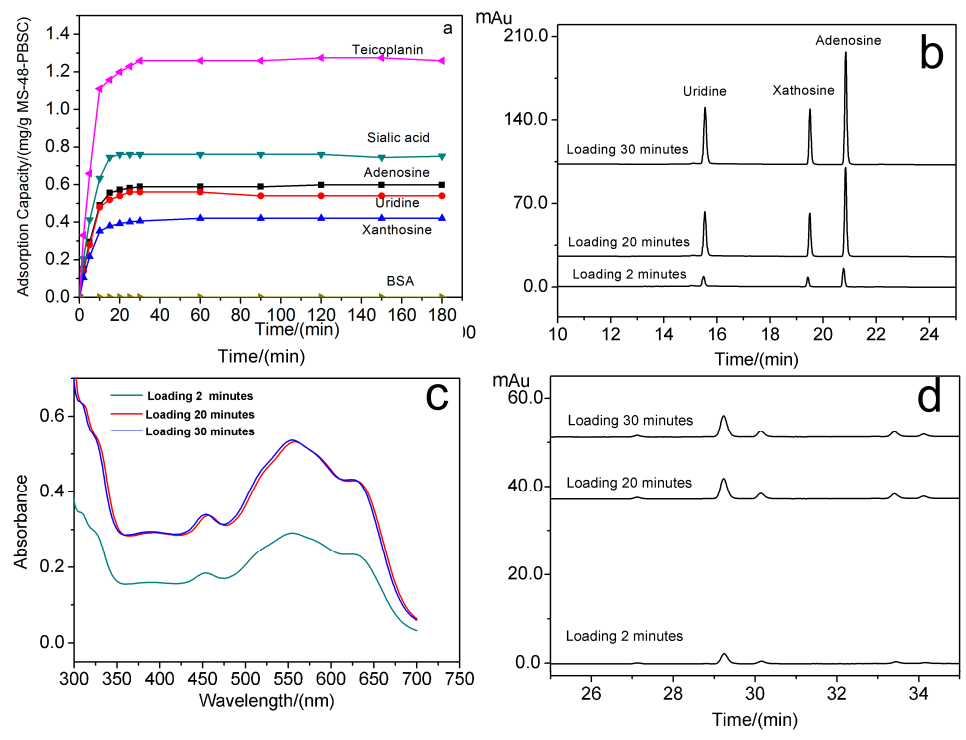

Figure 6. (a) Adsorption capacity at different loading times for uridine, xanthosine, adenosine, sialic acid, teicoplanin, and bovine serum albumin; (b) High-performance liquid chromatograph of the elution of xanthosine, uridine, and adenosine with different loading times; (c) Ultraviolet absorption spectrum of eluted sialic acid with different loading times; (d) High-performance liquid chromatograph of the elution of teicoplanin with different loading times. 
We applied a commercial magnetic nanoparticle prepared by coating a boron-based polymer onto the outer layer of the magnetic core for comparison. The maximum adsorption amount of commercial nanoparticle reached $0.36,0.48,0.51,0.54$, and $1.02 \mathrm{mg} \cdot \mathrm{g}^{-1}$ for adenosine, xanthosine, uridine, sialic acid, and teicoplanin, respectively. These results showed that the adsorption capacity of MS-48-PBSC was similar to that of the commercial material.

\subsection{Selectivity Investigation}

We also selected BSA as biological macromolecule interference and guanine, uric acid, and xanthine as small biological molecule interferences. The selectivity test results showed the MS-48-PBSC exhibited no adsorption toward BSA (Figure 6a) because BSA cannot enter the channel of MS-48-PBSC owing to the size exclusion effect. This outcome implied that the small pore entrance size of approximate $4.2 \mathrm{~nm}$ enabled the exclusion of macromolecule proteins.

The HPLC of the supernatants of uridine, xanthosine, adenosine, guanine, uric acid, and xanthine during loading, washing, and elution are shown in Figure 7. The small biological molecule interferences of guanine, uric acid, and xanthine without cis-diol structures existed in the loading and washing supernatants but were absent in the elution solution. Thus, the interferences were weakly adsorbed by the porous structure and easily desorbed during the washing step. By contrast, the targets of uridine, xanthosine, and adenosine with cis-diol structures were detected in the elution solution but not in the loading and washing supernatants. Thus, the materials exhibited high selectivity toward cis-diol structures due to the complex reaction. High selectivity was also derived from the mesoporous structure exclusion. This feature is beneficial for the application of MS-48-PBSC in complex biological samples, such as blood, urine, and tissue.

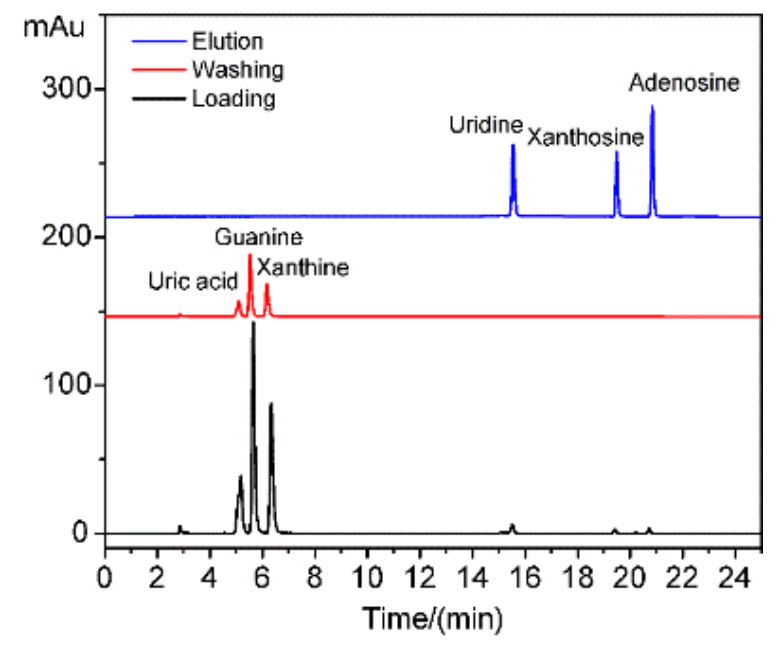

Figure 7. Selectivity toward uridine, xanthosine, adenosine, guanine, uric acid, and xanthine.

\subsection{Recovery and Enrichment Times}

The recovery of the test objects in Section 2.4 enriched by the MS-48-PBSC was investigated and the results are listed in Table 1. The recovery of three concentration levels of uridine, xanthosine, adenosine, sialic acid and teicoprazole were 71.8-114.1\% with RSD 1.5-8.6\%. The results suggested that the MS-48-PBSC could be used for separation and enrichment of cis-diol-containing compounds.

The concentrations of the five model compounds were increased after enrichment by MS-48-PBSC. The enrichment factor reached 10,11, 9, 10, and 9 corresponding to uridine, xanthosine, adenosine, sialic acid and teicoplanin respectively. After enrichment, the detection limits of cis-diol-contaning substances were decreased. 
Table 1. Recovery of the uridine, xanthosine, adenosine, sialic acid, and teicoplanin.

\begin{tabular}{ccccc}
\hline Sample & The Added Amount $(\boldsymbol{\mu g})$ & The found Amount $(\boldsymbol{\mu g})$ & Recovery $(\mathbf{\%})$ & RSD $(\%, \boldsymbol{n}=\mathbf{3})$ \\
\hline \multirow{3}{*}{ Uridine } & 0.533 & 0.407 & 76.4 & 4.4 \\
& 5.421 & 4.315 & 79.6 & 6.5 \\
Xanthosine & 20.09 & 16.19 & 80.6 & 5.7 \\
& 0.521 & 0.374 & 71.8 & 4.4 \\
& 5.222 & 3.765 & 72.1 & 1.5 \\
Adenosine & 20.31 & 14.97 & 73.7 & 5.7 \\
& 0.493 & 0.423 & 85.9 & 4.4 \\
& 5.279 & 4.413 & 83.6 & 6.5 \\
Sialic acid & 20.20 & 18.01 & 89.2 & 5.7 \\
\hline \multirow{3}{*}{ Teicoplanin } & 0.516 & 0.524 & 101.6 & 8.4 \\
& 5.178 & 4.287 & 82.8 & 6.7 \\
& 20.36 & 19.73 & 96.9 & 7.7 \\
\hline
\end{tabular}

\subsection{Comparative Results for the Different Methods}

The preparation method for MS-48-PBSC proposed in this paper has advantages in minimization of processing time and solvent consumption compared to those of heterogeneous reaction products containing at least two steps. This was attributed to the one-step preparation approach of MS-48-PBSC reduce the posttreatment steps. In extraction and enrichment processes, the MSPE method only used an external magnetic field for separation and enrichment. On one hand, it reduced the costs of the solid-phase extraction cartridge. On the other hand, the solvent usage of $1 \mathrm{~mL}$ for MS-48-PBSC method was less than that of the SPE method with at least 3-5 mL. Therefore, the MS-48-PBSC was more promising than the nanomaterials prepared with numerous heterogeneous reaction steps for extraction and enrichment of cis-diol-containing compounds.

\section{Materials and Methods}

$\mathrm{FeCl}_{3}(99.9 \%)$, ethylene glycol (98\%), and sodium acetate (99.9\%) were obtained from Sinopharm Chemical Reagent Co. Ltd., Shanghai, China. Pluronic polyethylene glycol-polylactic acid (PEG4000-PLA800 (P848)) was obtained from Jinan Daigang Co. Ltd., Jinan, China; tetraethoxysilane (TEOS, 98\%), triethoxysilane (97\%), pinacol (99\%) and 4-vinyl phenylboronicacid (98\%) bovine serum albumin $(98 \%)$, sialic acid $(98 \%)$, teicoplanin (98\%), uridine, xanthosine, adenosine, guanine, uric acid, and xanthine standards were obtained from Energy Chemica, Shanghai, China; pyrocatechol $(99.9 \%)$, hydroquinone $(99.9 \%)$, and trifluoroacetic acid were obtained from Shanghai Ling Feng Chemical Reagent Co. Ltd., Shanghai, China; Karstedt's catalyst was obtained from Alfa Aesar, Shanghai, China, commercial phenylboron modified magnetic nanomaterials were obtained from Enriching Biotechnology Ltd., Shanghai, China. All chemicals and solvents were used without further purification. $\mathrm{N}_{2}$ adsorption-desorption isotherms were measured at $196{ }^{\circ} \mathrm{C}$ using ASAP 2010 gas adsorption apparatus (Micromeritics Instrument Co., Norcross, GA, USA). Field emission scanning electron microscopy (FE-SEM) was performed on an FEI Magellan 400 electron microscope (Hillsboro, OR, USA). Transmission electron microscopy (TEM) images were taken with a JEOL JEM-2100 microscope operated at $200 \mathrm{kV}$ (Tokyo, Japan). The magnetic properties were determined with a physical property measurement system (PMS-9T (EC-II), Quantum Design Company of USA, San Diego, CA, USA). Fourier-transform infrared (FT-IR) spectrometry was carried out using a Nicolet 380 FT-IR spectrometer (Thermo Fisher Scientific, Waltham, MA, USA). Nuclear magnetic resonance (NMR) spectra was measured using an Agilent Technologies 400 MR (Santa Clara, CA, USA). Exact mass was measured with a Waters ACQUITY TQD Electrospray ionization mass spectrometry 
(ESI-MS) (Milford, MA, USA). Chromatography detection was carried out using a Shimadzu LC-20AT high-performance liquid chromatograph (Tokyo, Japan).

\subsection{Synthesis of Mesoporous Silica-Coated Magnetic Oxide (MS-48)}

Highly water-dispersible $\mathrm{Fe}_{3} \mathrm{O}_{4}$ nanospheres were prepared using the solvothermal reaction reported by Zhao et al. [19] $\mathrm{Fe}_{3} \mathrm{O}_{4} @ \mathrm{SiO}_{2}$ microspheres were prepared using sol-gel method [20]. The core-shell-structured MS-48 composites were prepared using the nonionic diblock copolymer Pluronic ${ }^{\circledR}$ PEG(4000)-PLA(800) (P48), developed by our laboratory [16] as template. MS-48 particles with $\sim 4.7 \mathrm{~nm}$ pore diameters were obtained after being refluxed in ethanol solution at $60^{\circ} \mathrm{C}$ for $48 \mathrm{~h}$ to remove the templates, and the reflux was repeated for three times.

\subsection{Synthesis of Mesoporous Boronic-Acid-Functionalized Magnetic Oxide (MS-48-PBSCP)}

4 -Vinyl benzene boronic acid pinacol ester was prepared according to the literature [21,22]. Specifically, a mixture of 4-vinyl phenylboronic acid $(0.5 \mathrm{~g})$ and pinacol $(0.44 \mathrm{~g})$ in $100 \mathrm{~mL}$ of dichloromethane in the presence of $20 \mathrm{~g}$ of $4 \mathrm{~A}$ molecular sieves was stirred at $40{ }^{\circ} \mathrm{C}$ for $3 \mathrm{~h}$ to give a near quantitative yield of 4 -vinyl benzene boronic acid pinacol ester. Analytical data for ${ }^{1} \mathrm{H}-\mathrm{NMR}$ of 4 -vinyl benzene boronic acid pinacol ester is given as ${ }^{1} \mathrm{H}-\mathrm{NMR}\left(\mathrm{CDCl}_{3}\right): \delta: 1.36(\mathrm{~s}, 12 \mathrm{H}), 5.31(\mathrm{~d}, 1 \mathrm{H}, J=8 \mathrm{~Hz})$, $5.83(\mathrm{~d}, 1 \mathrm{H}, J=16.0 \mathrm{~Hz}), 6.7(\mathrm{dd}, 1 \mathrm{H}), 7.41(\mathrm{~d}, 2 \mathrm{H}, J=8 \mathrm{~Hz}), 7.77(\mathrm{~d}, 2 \mathrm{H}, J=8 \mathrm{~Hz}) ;{ }^{13} \mathrm{C}-\mathrm{NMR}\left(\mathrm{CDCl}_{3}\right): 8: 140.17$, $136.84,134.98,125.46,114.79,114.77,83.71,77.00,24.82$; ESI-MS $(\mathrm{M}+\mathrm{H})^{+}: m / z=231.1$. Analytical data for ${ }^{1} \mathrm{H}-\mathrm{NMR}$ of the PBSCP is given as ${ }^{1} \mathrm{H}-\mathrm{NMR}\left(\mathrm{CDCl}_{3}\right): \delta: 0.91(\mathrm{~m}, 2 \mathrm{H}), 1.17(\mathrm{dt}, 9 \mathrm{H}, J=14 \mathrm{~Hz}$ and $J=6 \mathrm{~Hz}), 1.38(\mathrm{~s}, 12 \mathrm{H}), 2.71(\mathrm{~m}, 2 \mathrm{H}), 3.68(\mathrm{dm}, 6 \mathrm{H}), 7.22(\mathrm{~m}, 2 \mathrm{H}, J=8 \mathrm{~Hz}), 7.68(\mathrm{~d}, 2 \mathrm{H}, J=12 \mathrm{~Hz})$; ${ }^{13} \mathrm{C}-\mathrm{NMR}\left(\mathrm{CDCl}_{3}\right): \delta: 134.93,136.84,129.02,128.21,127.35,127.24,83.58,77.06,58.82,58.41,29.13,24.85$, $18.33,12.44$. ESI-MS $(\mathrm{M}+\mathrm{Na})^{+}: m / z=417.2$.

PBSCP was prepared according to the literature [23-25]. Specifically, $0.95 \mathrm{~g}$ of 4 -vinyl benzene boric acid pinacol ester and 300 ppm of Karstedt's catalyst were dissolved in $5 \mathrm{~mL}$ of drying $\mathrm{N}, \mathrm{N}$-dimethylformamide (DMF). After the mixture was activated for $20 \mathrm{~min}$ at $50{ }^{\circ} \mathrm{C}, 0.82 \mathrm{~g}$ of triethoxysilane was added dropwise to the solution and stirred for $5 \mathrm{~h}$ at $60^{\circ} \mathrm{C}$ under the protection of nitrogen. Finally, the DMF and excess triethoxysilane were removed under vacuum, and the nearly equivalent amount of PBSCP as a colorless oil was obtained.

MS-48 $(0.15 \mathrm{~g})$ and $0.2 \mathrm{~g}$ of $\mathrm{Na}_{2} \mathrm{CO}_{3}$ were dispersed in $20 \mathrm{~mL}$ of a mixed solution of toluene and DMF ( $v: v=2: 3)$. After ultrasonic treatment for $30 \mathrm{~min}, 0.5 \mathrm{~g}$ of PBSCP was added to the solution. The mixture was heated to $60{ }^{\circ} \mathrm{C}$ for $24 \mathrm{~h}$ with magnetic stirring under the protection of nitrogen. The products were collected with a magnet and washed twice by DMF followed by three washings with toluene. Finally, product MS-48-PBSCP was dried at $60^{\circ} \mathrm{C}$ overnight.

\subsection{Removal of the Pinacol Protecting Group from the MS-48-PBSCP}

Prior to extraction, MS-48-PBSCP was used to remove the pinacol-protecting group. In brief, $100 \mathrm{mg}$ of MS-48-PBSCP was added into $3 \mathrm{~mL}$ of $1 \mathrm{~mol} \mathrm{~L}^{-1}$ formic acid and methanol mix solution $(v: v=1: 1)$ for $30 \mathrm{~min}$ of sonication dispersion and vortex mixing. After isolation using a magnet, the supernatant with the pinacol protecting group was abandoned. Finally, the unprotected magnetic nanoparticle product MS-48-PBSC was washed with $3 \mathrm{~mL}$ of $50 \%$ methanol solution several times and $1 \mathrm{~mL}$ of phosphate-buffered saline (PBS) $(\mathrm{pH}=7.8)$ several times.

\section{4. pH Optimization of the Extraction and Desorption}

The $\mathrm{pH}$ optimization of the extraction and desorption were investigated according to the literature [16]. Typically, an adenosine solution at $20 \mu \mathrm{g} \cdot \mathrm{mL}^{-1}$ was selected as the test sample. In the loading process, $\mathrm{pH}$ of the adenosine solution was adjusted with PBS buffer or hydrochloric acid-tris buffer. After interacted with MS-48-PBSC nanoparticles for $30 \mathrm{~s}$ with the aids of ultrasonic and vortex mixing, the magnetic nanoparticles were isolated by a magnet and the supernatant was detected via HPLC. During the elution, adenosine-adsorbed MS-48-PBSC was eluted with different 
concentrations of formic acid solution and $40 \%$ methanol as elution solvent. The desorption time varies from $2 \mathrm{~min}$ to $3 \mathrm{~h}$, and the elutes were detected via HPLC.

\subsection{Enrichment of cis-Diol-Containing Compounds}

Adenosine, xanthosine, and uridine solutions were used as the typical nucleoside samples, sialic acid as the monosaccharide sample, teicoplanin as the antibiotic sample, and BSA as the macromolecular sample. Small biological molecules guanine, uric acid, and xanthine were selected as interferences samples. All samples were prepared as stock solutions at $1 \mathrm{mg} \cdot \mathrm{mL}^{-1}$.

For evaluation of the adsorption capacity of MS-48-PBSC, the above-mentioned cis-diol structure-containing samples except teicoplanin and BSA were formulated into $100 \mu \mathrm{g} \cdot \mathrm{mL}^{-1}$ (The concentration of teicoplanin was $200 \mu \mathrm{g} \cdot \mathrm{mL}^{-1}$ ) solutions with PBS $(\mathrm{pH}=7.8)$ buffer, respectively, and then $100 \mathrm{mg}$ MS-48-PBSC was added. The loading and elution processes were performed using the optimal conditions listed in Section 3.4. For the washing process, $3 \mathrm{~mL}$ of $\mathrm{PBS}(\mathrm{pH}=7.8)$ and acetonitrile solution $(v: v=90: 10)$ were added, and ultrasonic and magnetic separation steps were repeated. Finally, the supernatants were diluted 3-fold and detected using HPLC and UV-Vis spectrophotometer. Benzene boron modified commodity nanomaterials was activated with PBS buffer $(\mathrm{pH}=8.5)$ and $5 \%$ of cetyltrimethylammonium bromide in methanol $/ \mathrm{H}_{2} \mathrm{O}(v / v, 1: 1)$. The following operation was similar to the method used for MS-48-PBSC.

For evaluation of the selectivity to cis-diol-containing compounds, the $1 \mathrm{~mL}$ solution included all of the above nucleoside samples, and small biological molecules interferences samples at $1.7 \mu \mathrm{g} \cdot \mathrm{mL}^{-1}$ with $\mathrm{pH}=7.8$, and $60 \mathrm{mg}$ MS-48-PBSC was added. The eluate is finally concentrated to $100 \mu \mathrm{L}$. The loading, washing, and eluting processes were performed using the above optimal conditions. Then, the concentrated eluates were detected using HPLC and UV-Vis spectrophotometer.

For evaluation of the recovery and enrichment times, we used three nucleoside standards, sialic acid and teicoplanin as the test samples. All of them were mixed in the three quality levels at $0.5,5$, and $20 \mu \mathrm{g}$ respectively. Finally, the mixed cis-diol structure sample supernatants were detected using HPLC and UV-Vis spectrophotometer.

\subsection{HPLC and UV-Vis Methods}

The chromatographic column type was a VP-ODS C18 $(5 \mu \mathrm{m}, 4.6 \mathrm{~mm} \times 200 \mathrm{~mm}$, Shimadzu). The mobile phase was methanol: $0.2 \%$ acetic acid solution $(v: v=30: 70)$, the wavelength of diode array detector for HPLC was $260 \mathrm{~nm}$, the sample size was $10 \mu \mathrm{L}$, and the flow rate was $1 \mathrm{~mL} \cdot \mathrm{mL}^{-1}$.

BSA sample supernatants were diluted to 10:1 and detected using ultraviolet (UV)-visible (vis) spectrophotometry at $280 \mathrm{~nm}$.

Sialic acid sample supernatants were detected using a procedure reported in the literature [26]. In brief, $100 \mu \mathrm{L}$ of supernatant was added to $2 \mathrm{~mL}$ of a resorcinol-hydrochloric acid solution. After boiling for $30 \mathrm{~min}$, the mixture was treated in an ice-bath for $3 \mathrm{~min}$. Then, $4 \mathrm{~mL}$ of butyl acetate-butanol extract solution $(v: v=4: 1)$ was added to extract the derivative. The derivative was detected using UV-vis spectrophotometry at $580 \mathrm{~nm}$. The resorcinol-hydrochloric acid solution was prepared as follows: $20 \mathrm{~mL}$ of $8 \mathrm{~mol} \cdot \mathrm{L}^{-1} \mathrm{HCl}$ solution, $62.5 \mu \mathrm{L}$ of $0.1 \mathrm{~mol} \cdot \mathrm{L}^{-1} \mathrm{CuSO}_{4}$ solution, and $2 \mathrm{~mL}$ of $181.6 \mathrm{mmol} \cdot \mathrm{L}^{-1}$ resorcinol solution were mixed together and diluted with deionized water to $25 \mathrm{~mL}$.

Teicoplanin sample supernatants were detected according to the literature [27] by HPLC. The chromatographic column type was VP-ODS C18 (5 $\mu \mathrm{m}, 4.6 \mathrm{~mm} \times 200 \mathrm{~mm}$, Shimadzu). Mobile phase A was PBS ( $\mathrm{pH}=6.0$ ) and acetonitrile solution at 85:15 (v:v), while mobile phase B was PBS $(\mathrm{pH}=6.0)$ and acetonitrile solution at 30:70 (v:v). The diode array detector wavelength selection of HPLC was $260 \mathrm{~nm}$, the sample size was $10 \mu \mathrm{L}$, and the flow rate was $1 \mathrm{~mL} / \mathrm{min}$. The elution gradient conditions for the LC mobile phase were based on A/B from 100/0 (v/v) to 70/30 (v/v) at 32 min and $62.5 / 37.5(v / v)$ at $32 \mathrm{~min}$. The total chromatographic run duration was $35 \mathrm{~min}$. 


\section{Conclusions}

A new mesoporous material (MS-48-PBSC) for detecting cis-diol biological substances was developed. MS-48-PBSC with mesoporous silicon-coated $\mathrm{Fe}_{3} \mathrm{O}_{4} @ \mathrm{SiO}_{2}$ as the nucleus resulted applying in a material with a high specific surface area. The mesoporous size exclusion effect enabled the exclusion of macromolecular proteins, which is beneficial for complex biological samples. Magnetism allows the easy separation of MS-48-PBSC from a complex matrix through an external magnetic field and reuse of material. During material preparation, the one-step immobilization of phenylboronic acid groups on the mesoporous wall allowed for an easy, time-efficient operation. Moreover, the phenylboronic acid group-modified mesoporous material can function at a relatively low $\mathrm{pH}$ range and improve the binding selectivity and affinity toward cis-diol structures. The method can be further applied to enrich of sugar complexes with cis-diol structures in biological samples.

Acknowledgments: This research was supported financially by the National Natural Science Foundation of China (Nos. 81273481 and 81202493). This is an open project of the State Key Laboratory of Quality Research in Chinese Medicine (Macau University of Science and Technology, MUST-SKL-2016-06) funded by the Macao Science and Technology Development Fund, Macau special administrative region.

Author Contributions: P.H. M.Z. and H.F. conceived and designed the experiments; H.F. and J.H. performed the experiments and wrote the paper; H.Z., and Y.W. analyzed the data; M.Z. contributed to the writing of the paper.

Conflicts of Interest: The authors declare no conflicts of interest.

\section{References}

1. De Leoz, M.L.A.; An, H.J.; Kronewitter, S.; Kimb, J.; Beecroft, S.; Vinall, R.; Lebrilla, C. Glycomic Approach for Potential Biomarkers on Prostate Cancer: Profiling of N-Linked Glycans in Human Sera and pRNS Cell Lines. Dis. Markers 2008, 25, 243-258. [CrossRef] [PubMed]

2. Mccaffrey, A.P.; Meuse, L.; Pham, T.T.T.; Conklin, D.S.; Hannon, G.J.; Kay, M.A. RNA interference in adult mice. Nature 2002, 418, 38-39. [CrossRef] [PubMed]

3. Vermassen, T.; Van, P.C.; Lumen, N.; Decaestecker, K.; Vanderschaeghe, D.; Callewaert, N.O.; Villeirs, G.; Hoebeke, P.; Van Belle, S.; Rottey, S.; et al. Urinary prostate protein glycosylation profiling as a diagnostic biomarker for prostate cancer. Prostate 2015, 75, 314-322. [CrossRef] [PubMed]

4. Chen, S.C.; Kontoyiannis, D.P. New molecular and surrogate biomarker-based tests in the diagnosis of bacterial and fungal infection in febrile neutropenic patients. Curr. Opin. Infect. Dis. 2010, 23, 567-577. [CrossRef] [PubMed]

5. Kyselova, Z.; Mechref, Y.; Kang, P.; Goetz, J.A.; Dobrolecki, L.E.; Sledge, G.W.; Novotny, M.V. Breast cancer diagnosis and prognosis through quantitative measurements of serum glycan profiles. Clin. Chem. 2008, 54, 1166-1175. [CrossRef] [PubMed]

6. Liu, N.; Dai, Q.; Zheng, G.; He, C.; Parisien, M.; Pan, T. N(6)-methyladenosine-dependent RNA structural switches regulate RNA-protein interactions. Nature 2015, 518, 560-564. [CrossRef] [PubMed]

7. Chen, X.; Wang, Q. Solid Phase Extraction Technology and Application, 1st ed.; Science Press: Beijing, China, 2010; pp. 28-31. ISBN 9787030259691.

8. Xu, Y.; Wu, Z.; Zhang, L.; Lu, H.; Yang, P.; Webley, P.A.; Zhao, D. Highly specific enrichment of glycopeptides using boronic acid-functionalized mesoporous silica. Anal. Chem. 2009, 81, 503-508. [CrossRef] [PubMed]

9. Fontanals, N.; Marcé, R.M.; Borrull, F.; Cormack, P.A.G. Mixed-mode ion-exchange polymeric sorbents: Dual-phase materials that improve selectivity and capacity. TrAC Trends Anal. Chem. 2010, 29, 765-779. [CrossRef]

10. Li, D.; Chen, Y.; Liu, Z. Boronate affinity materials for separation and molecular recognition: Structure, properties and applications. Chem. Soc. Rev. 2015, 44, 8097-8123. [CrossRef] [PubMed]

11. Niu, H.Y.; Cai, Y.Q.; Shi, Y.L.; Wei, F.S.; Mou, S.F.; Jiang, G.B. Cetyltrimethylammonium bromide-coated titanate nanotubes for solid-phase extraction of phthalate esters from natural waters prior to high-performance liquid chromatography analysis. J. Chromatogr. A 2007, 1172, 113-120. [CrossRef] [PubMed] 
12. Yao, N.; Chen, H.M.; Lin, H.Q.; Deng, C.H.; Zhang, X.M. Enrichment of peptides in serum by $C(8)$-functionalized magnetic nanoparticles for direct matrix-assisted laser desorption/ionization time-of-flight mass spectrometry analysis. J. Chromatogr. A 2008, 1185, 93-101. [CrossRef] [PubMed]

13. Sha, Y.F.; Deng, C.H.; Liu, B.Z. Development of $C_{18}$-functionalized magnetic silica nanoparticles as sample preparation technique for the determination of ergosterol in cigarettes by microwave-assisted derivatization and gas chromatography/mass spectrometry. J. Chromatogr. A 2008, 1198, 27-33. [CrossRef] [PubMed]

14. Wang, Y.; Liu, M.; Xie, L.; Fang, C.; Xiong, H.; Lu, H. Highly efficient enrichment method for glycopeptide analyses: Using specific and nonspecific nanoparticles synergistically. Anal. Chem. 2014, 86, 2057-2064. [CrossRef] [PubMed]

15. Zhou, W.; Yao, N.; Yao, G.; Deng, C.; Zhang, X.; Yang, P. Facile synthesis of aminophenyl boronic acid-functionalized magnetic nanoparticles for selective separation of glycopeptides and glycoproteins. Chem. Commun. 2008, 43, 5577-5579. [CrossRef] [PubMed]

16. Li, H.; Shan, Y.; Qiao, L.; Dou, A.; Shi, X.; Xu, G. Facile synthesis of boronate-decorated polyethyleneiminegrafted hybrid magnetic nanoparticles for the highly selective enrichment of modified nucleosides and ribosylated metabolites. Anal. Chem. 2013, 85, 11585-11592. [CrossRef] [PubMed]

17. Fu, H.; Chen, Y.Q.; Zhang, H.Y.; Wang, Y.R.; Zhang, M.; Hu, P. Synthesis of Mesoporous Silica-Coated Magnetic Nanocomposites Using Polyethylene Glycol-Polylactic Acid as a New Template. J. Nanosci. Nanotechnol. 2017, 17, 3077-3083. [CrossRef]

18. Wang, H.; Bie, Z.; Lü, C.; Liu, Z. Magnetic nanoparticles with dendrimer-assisted boronate avidity for the selective enrichment of trace glycoproteins. Chem. Sci. 2013, 4, 4298-4303. [CrossRef]

19. Li, W.; Yue, Q.; Deng, Y.; Zhao, D. Ordered mesoporous materials based on interfacial assembly and engineering. Adv. Mater. 2013, 25, 5129-5152. [CrossRef] [PubMed]

20. Yang, J.; Zhang, F.; Li, W.; Gu, D.; Shen, D.; Fan, J.; Zhao, D. Large pore mesostructured cellular silica foam coated magnetic oxide composites with multilamellar vesicle shells for adsorption. Chem. Commun. 2014, 50, 713-715. [CrossRef] [PubMed]

21. Kaupp, G.; Naimi-Jamal, M.R.; Stepanenko, V. Waste-free and facile solid-state protection of diamines, anthranilic acid, diols, and polyols with phenylboronic acid. Chem. A Eur. J. 2003, 9, 4156-4161. [CrossRef] [PubMed]

22. Chan, D.M.; Monaco, K.L.; Li, R.; Bonne, D.; Clark, C.G.; Lam, P.Y.S. Copper-promoted C-N and C-O bond cross-coupling with phenyl and pyridylboronates. Tetrahedron Lett. 2003, 34, 3863-3865. [CrossRef]

23. Pelton, R.; Cui, Y.; Zhang, D.; Chen, Y.; Thompson, K.L.; Armes, S.P.; Brook, M.A. Facile phenylboronate modification of silica by a silaneboronate. Langmuir 2013, 29, 594-598. [CrossRef] [PubMed]

24. Matyjaszewski, K.; Miller, P.J.; Shukla, N.; Immaraporn, B.; Gelman, A.; Luokala, B.B.; Pakula, T. Polymers at interfaces: Using atom transfer radical polymerization in the controlled growth of homopolymers and block copolymers from silicon surfaces in the absence of untethered sacrificial initiator. Macromolecules 1999, 32, 8716-8724. [CrossRef]

25. Weng, W.W.; Chen, R.M.; Luh, T.Y. Palladium-catalyzed hydrosilylation of silyl-substituted butadienes. J. Heteroat. Chem. 1995, 6, 15-18. [CrossRef]

26. Chinese Pharmacopoeia of China Convention. Chinese Pharmacopoeia 3, 10th ed.; Science Press: Beijing, China, 2015; pp. 3102-3103. ISBN 9787506765299.

27. Chinese Pharmacopoeia of China Convention. Chinese Pharmacopoeia 2, 10th ed.; Science Press: Beijing, China, 2015; pp. 1252-1253. ISBN 9787506765299.

Sample Availability: Sample of the compound MS-48-PBSC is available from the authors. 